\title{
Desflurane anesthesia and postoperative cognitive function
}

\author{
Fang Ding $^{1,2} \cdot$ Limin Zheng ${ }^{1} \cdot$ Tao Luo ${ }^{1}$
}

Received: 6 March 2015 / Accepted: 8 March 2015 / Published online: 28 March 2015

(C) Japanese Society of Anesthesiologists 2015

\section{To the Editor:}

We read with interest the recent publication by Tachibana et al. [1]. The authors suggested that cognitive function $24 \mathrm{~h}$ after surgery was significantly improved in patients with desflurane anesthesia. There seem to be several problems that we should pay attention to.

First, patient-related factors including history of alcohol abuse, a lower educational level, and intraoperative factors such as deep anesthesia have been suggested with an increased risk for the development of POCD. These risk factors, which may have an influence on the results, should be considered in the subject enrollment and study design.

Second, the MMSE test quantitatively assesses cognitive impairment on a scale from 0 to 30 ; with a maximum score of 30 and a cut-off score of 24. A decrease in MMSE $>2$ points was usually considered clinically significant. Tachibana et al. reported a marginal intergroup difference in MMSE scores at $24 \mathrm{~h}$ after surgery $(p=0.048)$. The magnitude of change in MMSE scores within each anesthesia group was about only 1 point, plus all above the cutoff score of 24. These results, showing minimal changes in cognitive function assessed by MMSE, may be of no clinical significance.
Limin Zheng

496855048@qq.com

Tao Luo

496855048@qq.com

1 Department of Anesthesiology, Peking University Shenzhen Hospital, 1120 Lianhua Road, Shenzhen, China

2 Anhui Medical University, Hefei, China
In addition, the timing of testing is important as well. Cognitive function tested $24 \mathrm{~h}$ after surgery is greatly influenced by residual anesthetic drugs, surgical pain as well as pain control, and patient health status. Thus, the MMSE scores at only one time point shortly after the surgery may not be accurate enough for the assessment of postoperative cognitive function.

Taken together, it may be premature to draw any conclusion that desflurane anesthesia has better cognitive function.

Acknowledgments Supported by Nature Science Foundation of China Grant Numbers 81102863, 81271205.

\section{Reference}

1. Tachibana S, Hayase T, Osuda M, Kazuma S, Yamakage M. Recovery of postoperative cognitive function in elderly patients after a long duration of desflurane anesthesia: a pilot study. J Anesth. 2015. doi:10.1007/s00540-015-1979-y [Epub ahead of print] 